\title{
LA TRASCENDENCIA DE LA COMUNICACIÓN INTERCULTURAL EN LA SALUD PÚBLICA INFANTIL EN CCATCCA, QUISPICANCHIS, CUSCO, PERÚ
}

\section{THE TRA N S C E D E N C E O F IN T E R C U L T U R A L COMMUNICATION IN CHILD PUBLIC HEALTH IN CCATCCA, QUISPICANCHIS, CUSCO, PERU}

\section{${ }^{1}$ Gregorio Cusihuamán Sisa}

${ }^{2}$ Angélica Chalco Benavente

\section{Resumen}

El rol social de la Comunicación intercultural radica en analizar la problemática social; por ello, el objetivo de este artículo busca mostrar su importancia en el desarrollo de programas sociales referidos a la salud pública, más aún si en los años recientes los resultados son adversos en cuanto a la desnutrición infantil y la anemia en niños del distrito de Ccatcca. La metodología es de tipo descriptiva, con diseño no experimental transeccional de campo, asumiendo tres niveles estratégicos de muestreo, una revisión del contexto social, análisis de información secundaria y finalmente el trabajo de campo. Los resultados más significativos demuestran que los rasgos culturales generan barreras comunicacionales, las cuales terminan perjudicando el objetivo central de los programas sociales de Salud Pública; sobre todo cuando quienes dirigen los programas desconocen el idioma predominante del distrito y, peor aún, de la lógica connotativa del quechua como idioma; siendo esta una disyuntiva racional al momento de elaborar la papelería informativa, lo que termina convirtiéndose en barreras comunicacionales interculturales. E1 85\% de la población habla quechua como lengua natural; sin embargo, solo el 14\% del personal de salud conoce el quechua, situación que sin duda se constituye en una barrera al momento de tratar con el paciente y mucho más durante la campaña de salud contra la anemia.

Palabras Clave: Barreras comunicacionales, Campañas de Salud Pública, interculturalidad.

\begin{abstract}
The social role of intercultural communication lies in analyzing social problems; therefore, the objective of this study seeks to show the importance of intercultural communication in the development of social programs related to public health; even more if in recent years the results are adverse as to child malnutrition and anemia in children of the district of Ccatcca. The methodology is descriptive, with a non-experimental transectional field design, assuming three strategic levels of sampling, a review of the social context, analysis of secondary information and, finally, field work. The most significant results show that cultural traits generate communication barriers that end up damaging the central objective of social programs of Public Health; especially when those who run the programs are unaware of the predominant language of the district, and even worse of the connotative logic of Quechua as a language; being this, a rational disjunction at the time of preparing the information, which ends up becoming intercultural communication barriers. $85 \%$ of the population speaks Quechua as first language; however, only 14\% of the health staff knows Quechua, situation that undoubtedly constitutes a barrier when dealing with the patient and much more during the health campaign against anemia.
\end{abstract}

Keywords: Communication barriers, interculturality, public Health Campaigns.

${ }^{1}$ Universidad Nacional de San Agustín de Arequipa. Perú-Arequipa. E-mail: gcusihuaman@unsa.edu.pe ${ }^{2}$ Universidad Nacional de San Agustín de Arequipa. Perú-Arequipa. E-mail: rosabe@hotmail.com 


\section{INTRODUCCIÓN}

En los años recientes, a raíz de un largo abandono del Estado, sobre todo en centros poblados rurales, se ha considerado programas sociales con presupuestos importantes cuya finalidad es atender sectores vulnerables; sin embargo, los resultados no corresponden a los esfuerzos económicos del gobierno peruano. Nuestra preocupación radica en identificar qué aspectos han sido incidentales en ese "fracaso", desde una perspectiva comunicacional, sabiendo que los recursos son limitados.

La comunicación intercultural implica abordar situaciones de encuentro, contacto o acciones conjuntas entre individuos de distintas culturas, definiendo algunos conceptos relacionados con esta y asimismo explicando las razones que denotan su importancia, sus principios básicos, su finalidad, los objetivos y espacios donde se desarrolla (Parra Crespo, 2010, p. 16). En tal sentido, dicha comunicación responde a las capacidades humanas de los actores como servidores y/o prestadores del servicio de salud pública, en una relación armónica entre el establecimiento de salud (personal asistencial) y las madres que tienen hijos menores de 5 años.

Uno de los problemas de mayor interés para la Salud Pública es la alta tasa de desnutrición crónica y anemia en niños menores de 5 años de edad, más aún cuando la madre no reconoce los signos de desnutrición del niño. Por tanto, es importante apostar por la alimentación y nutrición, especialmente en las niñas y niños, debido a que en esta etapa estimulan el desarrollo cerebral relacionado a la capacidad de asimilar el conocimiento, interactuar con su entorno, su capacidad motora y de adaptación a nuevos ambientes y personas, entre otros. El que un niño esté o no bien alimentado, durante los primeros años de vida, puede tener un efecto contundente en su salud a futuro.

En los programas de salud únicamente se tomaron en cuenta los mensajes en quechua - tal cual se percibe en el anexo-, mas no fueron considerados otros rasgos interculturales como las identidades, tradiciones, religiosidad; en consecuencia, se puede deducir que obviaron barreras comunicacionales interculturales significativas.

En alusión estricta a nuestra investigación referida a la lengua como elemento cultural central, la interculturalidad tendría que entenderse en la magnitud propuesta por Degregori Caso (2013), quien señala:

En pleno proceso de globalización, conforme se intensifican las comunicaciones, los desplazamientos y vínculos entre todos los pueblos y Estados del mundo, se fortalecen al mismo tiempo las identidades y las lealtades locales especialmente aquellas conformadas alrededor de la lengua, la religión, las tradiciones, las formas de organización social, es decir alrededor de la cultura y la historia (p. 349).

\section{ANTECEDENTES}

La Comunicación Intercultural incide directamente en los programas de Salud Pública, y para demostrarlo hemos considerado elementos del idioma así como algunas características socioeconómicas de las madres.

En el análisis de la lengua española se tiene varias acepciones que certifican su claridad de enrostrar el mensaje, tanto en la interrelación oral como en la simbólica, lo que en este caso fortalece la idea del mensaje como elemento generador de confianza mutua; no obstante, en la lógica del idioma quechua queda claramente como un elemento disfuncional, a la luz de la explicación del Dr. Mannheim, pues la visión de análisis del quechua no responde a la lógica tradicional del español (Sánchez Arroba, 2016). A ello debe sumarse, como una característica sociocultural actual —según Tannen (1999, p. 248) - que cada cultura dispone de sus propios medios para expresar desacuerdo y conflicto. Aquellas sociedades que hacen hincapié en la armonía desarrollan actividades que pueden sorprender (e iluminar) a las que tienden a fomentar la oposición.

Con respecto a Ccatcca, esta se ubica en una región rural y, como señala Cavero Carrasco 
(2011), debido a la pobreza que padece se convierte en prioridad básica, siendo todo lo demás accesorio y complementario (p. 115). En este análisis resulta valioso, además, tener en cuenta el aspecto cultural, en tal sentido Clifor (2003) sostiene lo siguiente: "somos animales incompletos o inconclusos que nos complementamos por obra de la cultura, y no por obra de cultura en general sino por formas en alto grado particulares de ella" (p. 55). De allí que consideremos relevante, en esta investigación, aquello que Hall (2010) señala como "el sistema y las convenciones de la representación, los códigos de sus lenguajes y cultura, que los equipan con un saber hacer cultural que a su vez les posibilita funcionar como sujetos culturalmente competentes" ( $p$. $450)$.

A esto se suma la incapacidad de los profesionales de la salud tanto en el habla como en el entendimiento del idioma natural del lugar donde ejercen su labor; lo cual, específicamente, repercute en los programas de difusión en el centro poblado de Ccatcca volviéndolos deficientes e ineficaces.

Un aporte interesante a este entender lo da Fierro (2000), quien afirma que la creación del lenguaje oral antecedió al lenguaje escrito y que ambos surgieron del desarrollo del pensamiento humano y sus diferentes estadios evolutivos; así también de la conciencia paulatina desarrollada en el hombre de cubrir sus necesidades de cualquier tipo, incluidas desde luego las de comunicación o, en su defecto, de la información. Esto viene a colación en el sentido de que si bien la población puede ser analfabeta en su mayoría, el programa de difusión resulta incluso más determinante en su proceso de elaboración y en la capacidad de quienes conducen su difusión.

\section{METODOLOGÍA}

Considerando que el problema se centra en identificar la trascendencia de la Comunicación Intercultural en la Salud Pública infantil, el enfoque metodológico responde a una investigación de tipo descriptiva, en la medida que define rasgos, cualidades o atributos de la población objeto de estudio; con diseño no experimental transeccional de campo.

Durante el proceso de investigación e indagación, se tuvo en cuenta tres niveles definidos claramente: en el primer nivel se realizó una revisión documental de fuentes secundarias, tanto bibliográfica como de la normatividad legal correspondiente; en el segundo nivel se determinó a través de una guía de observación el entorno de la institución de salud del distrito y las características del personal de salud, así también de las organizaciones de interés del centro de salud; y en el tercer nivel se destacó la información primaria, en base a entrevistas al personal asistencial y encuestas realizadas a los pobladores.

El ámbito de estudio se ubica en la región Cusco, en la provincia de Quispicanchis, distrito de Ccatcca, específicamente en su puesto de salud, el cual se encuentra a una altitud de $3.700 \mathrm{msnm}$. Dicho distrito fue creado mediante Ley del 2 de enero de 1825 , firmada por el libertador Simón Bolívar.

El período de la investigación abarca desde junio del año 2015 hasta marzo de 2016, temporada posterior a campañas de salud en el distrito, teniendo por base información estadística de los establecimientos de salud, donde no se ha notado mejoría estadística en los problemas de anemia y desnutrición infantil, como consecuencia de los programas implementados por el sector.

El año 2013, Ccatcca contaba con una población total de 17264 habitantes, de los cuales $8660(50,16 \%)$ son varones y 8604 $(49,84 \%)$ mujeres. Asimismo se tiene una data de 2359 niños de 0 a 4 años en todo el distrito, el cual está conformado por 24 comunidades y centros poblados.

Según el informe del Centro de Salud de Ccatcca solo acude aproximadamente el $30 \%$ de los niños, lo cual significa que 708 niños son quienes logran tener control infantil. Esta población infantil registrada en el centro poblado de Ccatca, se tomó como universo. 
La encuesta personalizada se realizó al 10\% de las madres, de forma aleatoria simple en razón a que todas ellas cumplen con la única condición de tener hijos menores de 5 años de edad que acuden cotidianamente a dicho centro de salud. Conocedores de la idiosincrasia del habitante de la zona — cabe resaltar que la población es sumamente renuente a conversar con personas extrañas y más cuando se les hace encuestas-, esperamos las respuestas de un $50 \%$, aproximadamente, porcentaje que se tomó como muestra representativa y valedera para la investigación.

\section{RESULTADOS}

En el análisis del contexto se consideró características socioeconómicas, edad, ocupación, grado de educación, estado civil, productos que cultiva. Esto en razón de que se trata de un sector rural y las características de lo que produce constituyen la economía básica de cada familia.

En primer término se describe la edad de las madres (ver Tabla 1 y Figura 1), pues resulta relevante detalles personales que pueden trascender en las decisiones de alimentación de los niños.

Tabla 1. Edad de Madres

\begin{tabular}{|c|c|c|}
\hline Años & Cantidad & $\%$ \\
\hline Hasta 19 años & 10 & 14,29 \\
\hline De 20 A 24 años & 16 & 22,86 \\
\hline De 25 A 29 años & 15 & 21,43 \\
\hline De 30 A 34 años & 15 & 21,43 \\
\hline De 35 A 39 años & 10 & 14,29 \\
\hline De 40 A 45 años & 4 & 5,71 \\
\hline Total & 70 & 100 \\
\hline$\underset{\substack{21.43 \\
\%}}{14.29}$ & $\begin{array}{c}14.29 \\
-\% 22.86 \\
\Gamma^{\%}\end{array}$ & $\begin{array}{l}\text { hasta } 19 \\
\text { años } \\
\text { de } 20 \text { a } 24 \\
\text { años } \\
\text { de } 25 \text { a } 29 \\
\text { años }\end{array}$ \\
\hline
\end{tabular}

Figura 1. Edad de las Madres
Como en la mayoría de zonas rurales, la maternidad en Ccatcca se produce en mujeres bastante jóvenes, menores de 19 años, cuyo porcentaje es elevado y problemático si consideramos que todavía están en edad de estudiar y muchas de ellas no concluyen ni la primaria o la secundaria, menos aún han iniciado estudios superiores; abandonan sus estudios para dedicarse a cuidar a los hijos, realizar labores del hogar y trabajar junto con la pareja. En algunos casos la reproducción se da desde la etapa de la pubertad: situación que agrava las condiciones de la mujer, de la familia y de la sociedad en esta localidad.

En un mayor porcentaje la maternidad se desarrolla entre las mujeres de 20 a 24 años con $22,86 \%$ de las madres que asisten al Centro de Salud de Ccatca, es cierto que en esta etapa la mujer se considera en su mayoría de edad y en plenitud de tener hijos; sin embargo, las condiciones socioeconómicas no cambian, pues los niveles de pobreza siguen siendo los mismos.

Un grupo más consolidado que continúa en porcentaje está conformado por mujeres de 25 a $29(21,43 \%)$; a quienes siguen las madres de 30 a 34 con $43 \%$, cuyas capacidades y aprendizaje en la crianza es mejor y más responsable.

Con menor porcentaje está el grupo de madres de 35 a 39 años, que representan el 14, 29\% la misma cantidad de quienes son menores de 19 años.

Finalmente, se encuentra el grupo de las mayores de edad, las cuales van de los 40 a los 45 años, representando el $5,71 \%$, periodo en que se corre el riesgo de nacimiento de niños con alguna deficiencia física o mental; no obstante los riesgos que puedan darse, aun así se embarazan.

El embarazo a temprana edad trae consigo un mayor incremento de población, y con los escasos recursos económicos que disponen los padres no se puede atender a satisfacción la demanda de los niños.

Otro factor relevante resulta el nivel de educación de las madres, una mejor formación 
educativa podría ayudar en la toma de decisiones adecuadas en la alimentación de los niños.

Tabla 2. Grado de Educación

\begin{tabular}{lcc}
\hline Estudios & Cantidad & $\%$ \\
\hline Sin Estudios & 45 & 64,29 \\
Primaria & 25 & 35,71 \\
Secundaria & 0 & 0,00 \\
Superior & 0 & 0,00 \\
\hline Total & 70 & 100 \\
\hline
\end{tabular}

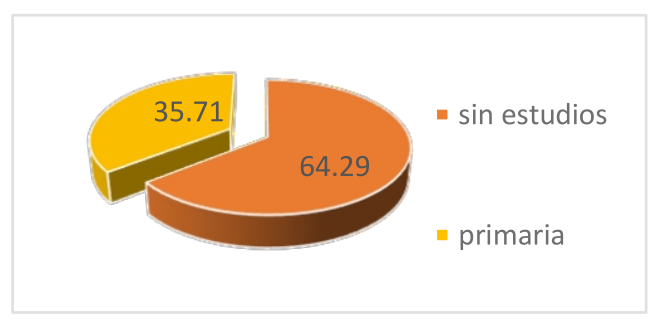

Figura 2. Grado de Educación

Como se puede apreciar en la tabla y la figura anteriores, el 64,29\% de las madres encuestadas manifestaron no tener educación o estudio alguno, y solo el 25\% ha alcanzado la educación primaria, lo cual demuestra claramente el desinterés por la educación de las mujeres en edad de estudiar que se han convertido en madres.

Por estas razones, podemos colegir y aseverar que el sistema educativo en Ccatca no ha cambiado sustancialmente y que una tarea pendiente es su completa renovación; asimismo, precisamos que los habitantes de este distrito necesitan desarrollar capacidades y habilidades para relacionarse con poblaciones de diferentes culturas que se sitúan en su entorno, evitando el aislamiento. Por lo tanto, urge una reforma educativa que apunte a solucionar verdaderamente los problemas educacionales, encare las deficiencias culturales y logre la inclusión social.

Para desarrollar las capacidades humanas necesitamos formar personas capaces de enfrentar las adversidades y retos en los diversos contextos de nuestro territorio, sobre todo en una sociedad del conocimiento en permanente cambio. Esta formación tiene que darse a lo largo de la vida, convirtiendo la etapa escolar en una oportunidad para lograr ese potencial en sus más altos niveles, situación que lamentablemente aún no se presenta en Ccatcca.

Niños y niñas, adolescentes y jóvenes necesitan aprender a lo largo de su educación básica las competencias necesarias para crecer como ciudadanos, desarrollarse a sí mismos y desenvolverse bien en el complejo mundo de hoy, así como para alcanzar las metas que se propongan como personas. Saber desenvolverse en los distintos escenarios y afrontar los desafíos.

Por otro lado, las tecnologías contemporáneas en el sector educación no llegan al distrito o no se sabe cómo utilizarlas. Los materiales educativos que se emplean constituyen un medio importante, cuyo propósito es facilitar el proceso de enseñanza-aprendizaje; sin embargo, pese a su importancia, estos recursos o herramientas pedagógicas todavía son elaborados por los propios profesores, con los escasos recursos económicos y materiales de la localidad.

En cuanto a la familia, esta permite la generación de un espacio donde se consolidan los roles asignados a sus integrantes con el comportamiento diario, allí los padres ejercen una influencia directa en la manifestación social de los hijos, aportando pautas, normas y modelos, sin que esto signifique que la familia sea en sí misma una manifestación espontánea de buenos o malos aprendizajes para los hijos en edad escolar.

La comunidad local, por su parte, se constituye en una fuente de numerosos aprendizajes, hábitos y costumbres, que otorga identidad cultural. De un lado está su historia y los hitos más significativos de su proceso de configuración y desarrollo. De otro lado, están sus personajes, individuos o grupos que han jugado o siguen jugando un rol importante en la vida de la comunidad. Se encuentran, asimismo, sus lugares y paisajes que por algún rasgo particular tienen un especial significado para sus habitantes. Finalmente, están sus actividades más distintivas, tanto las de orden económico como social y cultural. 
Tabla 3. Estado Civil

\begin{tabular}{lcc}
\hline Estado Civil & Cantidad & $\%$ \\
\hline Madre Soltera & 10 & 14,29 \\
Casada & 13 & 18,57 \\
Conviviente & 40 & 57,14 \\
Viuda & 4 & 5,71 \\
Divorciada & 3 & 4,29 \\
\hline Total & 70 & 100 \\
\hline
\end{tabular}

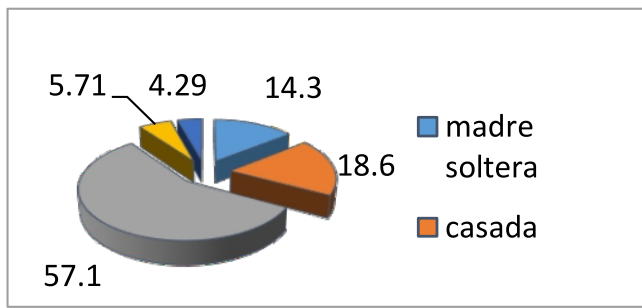

Figura 3. Estado Civil

Tal como se aprecia en la Tabla 3 y en la Figura 3 , el $57,14 \%$ de las mujeres opta por la convivencia y solo el $18,57 \%$ por el matrimonio. Lo cual demuestra que menos del $20 \%$ se preocupa por la unión legal y alrededor del $80 \%$ tienen vida con pareja, en su defecto con hijos que pueden resultar con menor atención frente a problemas.

Este tipo de libertad, que muestra la característica cultural del distrito, es completamente diferente al de las sociedades urbanas, debido a la cosmovisión de las poblaciones, tradiciones, costumbres - cultura-, de esos tiempos, las poblaciones del Perú profundo mantienen sus costumbres, su cultura.

Debido a esto, las diferentes disposiciones en materia de salud que se emanan desde la capital no son aceptadas por las diferentes culturas del país; por ello es importante llegar a estas comunidades y estructurar con sus habitantes las políticas, objetivos, proyectos, programas y acciones a realizar; solo así se podrá mejorar las condiciones de vida de toda la población, sin imposiciones ni modificación alguna de su cultura, por el contrario, debemos integrarnos unos a otros: esta debe ser nuestra fortaleza.
Tabla 4. Diversidad Idiomática

\begin{tabular}{lcr}
\hline Estudios & Cantidad & \multicolumn{1}{c}{$\%$} \\
\hline Quechua & 60 & 85,71 \\
Aymara & 0 & 0,00 \\
Castellano & 10 & 14,29 \\
Otros & 0 & 0,00 \\
\hline Total & 70 & 100 \\
\hline
\end{tabular}

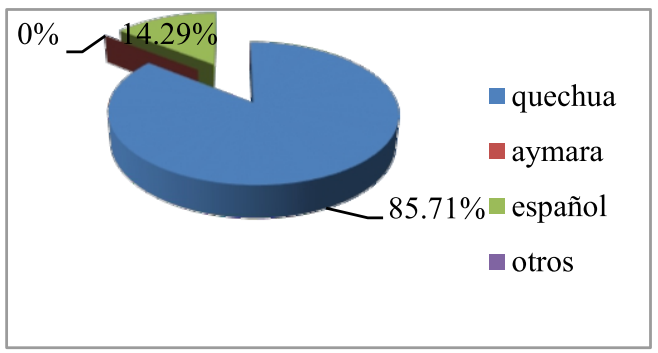

Figura 4. Diversidad idiomática

En relación al idioma que practican cotidianamente en el distrito de Ccatcca, conviene señalar antes algunos datos conceptuales que son pertinentes:

Idioma: "Del lat. Tardío. Se define como idioma a 'peculiaridad de estilo', 'lenguaje propio de un autor', m. Lengua de un pueblo o nación, o común a varios". (RAE, 2014)

Lengua: "Del lat. lingua, f. Sistema de comunicación verbal y casi siempre escrito, propio de una comunidad humana". (RAE, 2014)

Lenguaje: "m. Capacidad propia del ser humano para expresar pensamientos y sentimientos por medio de la palabra. Sistema de signos que utiliza una comunidad para comunicarse oralmente o por escrito. Sinónimos: idioma, lengua”. (RAE, 2014)

La Constitución Política del Perú, en su art. $48^{\circ}$, determina lo siguiente: "Son idiomas oficiales el castellano y, en las zonas donde predominen, también lo son el quechua, el aimara y las demás lenguas aborígenes, según la ley". 
La Ley $\mathrm{N}^{\circ} 29735$ promulgada el 02 de julio de 2011, en el Art. 3 sobre Definición de lenguas originarias, señala:

Para los efectos de la aplicación de la presente Ley, se entiende por lenguas originarias del Perú a todas aquellas que son anteriores a la difusión del idioma español y que se preservan y emplean en el ámbito del territorio nacional.

Asimismo, en el art. $23^{\circ}$ sobre alfabetización intercultural se afirma que "Los programas de alfabetización en zonas rurales andinas y amazónicas se implementan mediante la modalidad intercultural bilingüe".

Dentro de la población encuestada el $85,71 \%$ es quechua-hablante y solamente el 14, 29\% habla español. Esto significa que el quechua es la lengua materna hablada por la gran mayoría de la población y el desenvolvimiento cotidiano se realiza mediante la comunicación y comprensión de este idioma, situación que muchos citadinos no alcanzan a comprender y es que su mundo y la comprensión de este otro son diferentes. Los habitantes de este distrito ven con distintos ojos el desarrollo de su comunidad, es poco relevante el modernismo urbano, les da lo mismo; puesto que su inquietud gira en torno a la tierra que cultivan, a sus productos y a la posibilidad de alimentar a sus descendientes.

Todavía se escucha a algunos padres de familia decir a sus hijos: “¿para qué estudian si no les va a servir?, lo que tienen que hacer es trabajar su tierra y con ello podrán vivir". Afortunadamente son pocos, la gran mayoría ya se ha dado cuenta de que en pleno siglo XXI los jóvenes tienen que afrontar diversos desafíos en diferentes escenarios, y que el desarrollo actual va a una velocidad distinta a la del campo.

Actualmente, los padres son conscientes de que los niños, adolescentes y jóvenes ccatqueños necesitan y demandan aprender competencias a través de la Educación Básica Regular, y desarrollar habilidades necesarias para crecer como personas. Del mismo modo, deben desarrollar una profesión ya sea técnica o universitaria para integrarse a la actividad laboral que la sociedad demanda. Desenvolverse y desarrollarse a sí mismos resulta complejo en el mundo de hoy, sin embargo, ya se nota el esfuerzo que ponen los jóvenes para cambiar las condiciones sociales de su familia.

En ese sentido, el uso de la lengua materna y su continuidad son vitales para el ser humano, ya que le permite establecer comunicación con el conjunto de personas integrantes de su comunidad y esta con otras; así como la sociedad en su conjunto, puede también desarrollarse y avanzar en el tiempo.

La dedicación cotidiana de las madres de Ccatcca nos permite tener mejor perspectiva del caso:

Tabla 5. Ocupación

\begin{tabular}{lcr}
\hline Ocupación & Cantidad & \multicolumn{1}{c}{$\%$} \\
\hline Profesional & 0 & 0,00 \\
Ama de casa & 56 & 80,00 \\
Comerciante & 10 & 14,29 \\
Empleada Doméstica & 0 & 0,00 \\
Desempleada & 4 & 0,00 \\
\hline Total & 70 & 100 \\
\hline
\end{tabular}

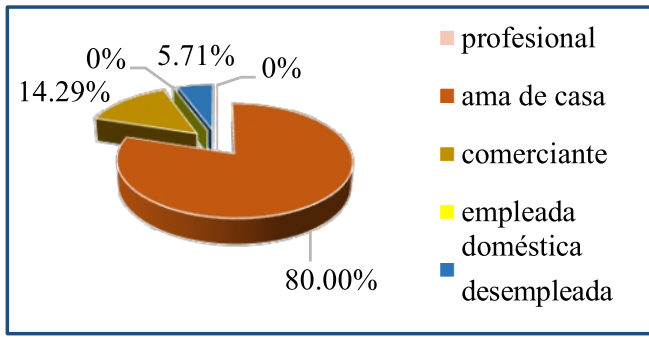

Figura 5. Ocupación

La tabla y el gráfico anteriores expresan la estructura de la población en cuanto a sus actividades laborales, el $80 \%$ de las mujeres encuestadas se dedica a las actividades del hogar, por tanto no contribuyen económicamente con el ingreso familiar; por otro lado, solo el $14,29 \%$ de las encuestadas tiene un ingreso económico más o menos permanente y mejora sus condiciones familiares. El desempleo alcanza al 5,71\% por cuanto puede considerarse bastante reducido. 
A esto debemos agregar que las mujeres que realizan labores como amas de casa, también realizan actividades de labranza en épocas en que la agricultura demanda mano de obra, con lo que su esfuerzo es el doble. Esto deteriora la esperanza de vida en las mujeres campesinas.

Tabla 6. Producción

\begin{tabular}{lcc}
\hline Productos & Cantidad & $\%$ \\
\hline Quinua, Tarwi & 10 & 14,29 \\
Papa, Maíz, Trigo & 40 & 57,14 \\
Cuy, Huevos, Verduras, & 12 & 17,14 \\
Otros & 8 & 11,43 \\
\hline Total & 70 & 100 \\
\hline
\end{tabular}

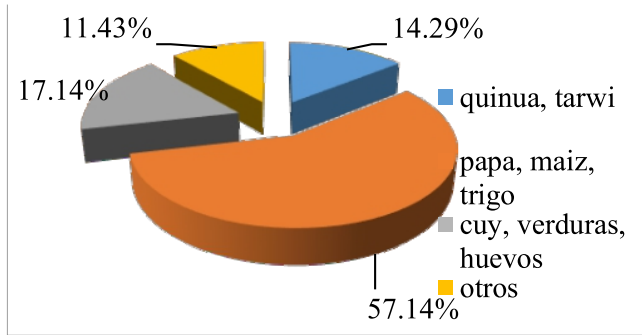

Figura 6. Producción

De acuerdo a la encuesta podemos afirmar que la producción de familias, en su mayoría, son tradicionales como la papa, el maíz, el trigo, con $57,14 \%$ siendo estos los alimentos básicos en su alimentación cotidiana que más se cultivan; seguidos de quinua y tarwi con el 14,29\%; y algunos productos de huerta para el consumo diario, los cuales no representan un peso suficiente como para tener el carácter de comercialización que posibilite ingresos económicos suficientes para la familia. En cuanto a lo pecuario, se aprecia mayormente la cría de animales domésticos que sirven para la alimentación familiar.

En Ccatcca, respecto a la producción agropecuaria, predomina lo agrario frente a lo pecuario; con relación a otro tipo de producción, manifiesta un nivel de ruralización elevado, es decir, con procesos y métodos tradicionales. Del mismo modo, es escasa la dotación de recursos naturales por la topografía geográfica en que se asentó la población
Ccatqueña, estas características determinan el tipo económico y de productividad y, a la vez, condicionan el grado de desarrollo agrario tanto como la vocación productiva de la zona en estudio.

La característica más resaltante del sector agropecuario en la zona, es la pequeña producción rural, la misma que involucra a toda la población Ccatqueña; puesto que el área urbana es reducida y las actividades comerciales y de transacción son todavía reducidas, todas ellas dependen de la actividad principal agraria.

La economía depende de los pequeños productores del campo, quienes son productores individuales o personales con pequeñas propiedades que provienen de la herencia que se ha sucedido de padres a hijos y continúa de esta manera, por lo que las parcelas cada vez son menores, esto obliga a que la mano de obra sea eminentemente familiar y no remunerada para poder acumular y solventar la demanda familiar.

Respecto al modo en que se adquiere el conocimiento para alimentar adecuadamente a sus hijos, en la siguiente tabla, como en la figura, se ilustra las respuestas de las encuestadas:

Tabla 7. ¿Quién le informó cómo alimentar a su hijo?

\begin{tabular}{lcc}
\hline Descripción & Cantidad & $\%$ \\
\hline Establecimiento de Salud & 16 & 22,86 \\
Promotor de Salud & 20 & 28,57 \\
Radio & 8 & 11,43 \\
Televisión & 0 & 0,00 \\
Otras madres & 26 & 37,14 \\
\hline Total & 70 & 100 \\
\hline \multicolumn{3}{|c|}{ - Establecimiento de } \\
\hline
\end{tabular}

Figura 7. ¿Quién le informó cómo alimentar a su hijo? 
A partir de las encuestas realizadas a las madres de familia, se observa que en primer lugar el $37,14 \%$ sabe cómo alimentar a su bebé porque pasan el conocimiento de madres a hijas, es la primera forma; con el $28,57 \%$ están los promotores de salud quienes informan cómo hacerlo, además por ser los más cercanos y porque hablan el mismo lenguaje; en tercer y penúltimo lugar, aprenden porque los médicos $\mathrm{y}$ enfermeras en los establecimientos de salud les dan esta información cuando realizan su control pre natal; en último lugar, están los medios de comunicación, debido a que el tema no es comercial ni redituable para los medios de comunicación o el periodismo.

Si bien el proceso de alimentación inicial del bebé se realiza intuitivamente y de manera natural a partir de su nacimiento, lo que la mayoría de las madres no sabe es que este proceso empieza con la propia concepción, de tal manera que todo aquello que ingiera la madre, o deje de hacerlo, influye directamente en la formación y crecimiento del feto.

En zonas rurales del Perú, existen varios parámetros que impiden una comunicación fluida por parte de los medios de comunicación, entre estos se encuentran los mismos aparatos, la energía, etc. por lo que la comunicación de mayor uso es la directa.

Otro detalle singular resultó a partir de la pregunta sobre qué síntomas reconocen las madres en un niño con anemia:

Tabla 8. Característica del niño con anemia

\begin{tabular}{|c|c|c|}
\hline Características & Cantidad & $\%$ \\
\hline Palidez & 15 & 15,43 \\
\hline Mareo & 14 & 20,00 \\
\hline No Juega & 12 & 17,14 \\
\hline Todos & 9 & 12,86 \\
\hline$\underline{\text { No Sabe }}$ & 20 & 28,57 \\
\hline Total & 70 & 100 \\
\hline 28 & & $\begin{array}{l}\square \text { palidez } \\
\square \text { mareo } \\
\text { no juega } \\
0 \% \text { fodos } \\
\text { no sabe }\end{array}$ \\
\hline
\end{tabular}

Figura 8. Características del niño con anemia
Se preguntó a las madres cuál es la característica que evidencia mejor a un niño con anemia, la respuesta más significativa es que no saben, el $28,57 \%$ de las madres desconoce cuáles son los síntomas; en segundo lugar tenemos con el $21,43 \%$ a las madres que dicen reconocer la anemia a través de la palidez, por cierto es uno de los síntomas más característicos; en tercer lugar, está el 20\% que manifiesta ser el mareo una de sus características más notorias, y en efecto es también uno de sus síntomas más conocidos; en cuarto lugar, tenemos al 17,14\% de madres que dicen no juega, le entra flojera, no hace ejercicio, lo que también es otro de los síntomas; finalmente, en quinto lugar se encuentra el $12,86 \%$ que dice reconocer todos los síntomas anteriores, lo cual es positivo.

Lo que nos preocupa sobremanera es el 28,57\% que no conoce los síntomas de una enfermedad que a simple vista se puede detectar; por ello, madres y padres deben recibir directamente y por folletos, con gráficos y dibujos, una información más amplia a través de su idioma quechua, con paciencia, usando ejemplos, fotografías, etc. No debe haber exclusión alguna, y de ser necesario se debe visitar a las familias casa por casa.

En referencia al tema de la anemia existe variedad de opiniones, y no todos los médicos coinciden con ellas. No obstante, se ha optado por el trabajo de Esther Martín, estudiante de medicina de la Universidad de Alcalá de Henares, revisado por el doctor Pablo Rivas, especialista en medicina interna; según nuestro parecer, el más completo. En dicho trabajo se señala lo siguiente:

El síndrome anémico lo forman un conjunto de signos y síntomas que ponen de manifiesto la disminución de la hemoglobina y el desarrollo del mecanismo de compensación. Los principales síntomas de la anemia son:

Palidez. Es uno de los signos más característicos de la anemia.

Astenia: constituye un síntoma general muy ligado a la anemia (el individuo anémico se siente "cansado").

Disnea: sensación subjetiva de falta de aire, se siente fatigado. 
Fatiga muscular: incluso con pequeños esfuerzos, no puede hacer ejercicios físicos. Manifestaciones cardiocirculatorias: taquicardia y palpitaciones que son constantes en caso de anemia moderada o intensa, especialmente cuando se instaura bruscamente.

Trastornos neurológicos: alteraciones de la visión. Cefaleas (dolor de cabeza).

Manifestaciones neuromusculares: consisten principalmente en cambio de la conducta, cefaleas, vértigos, trastornos visuales, insomnio, incapacidad para concentrarse y, ocasionalmente, desorientación.

Alteraciones del ritmo menstrual: la existencia de reglas abundantes (hipermenorrea) es causa frecuente de anemia en las mujeres jóvenes.

Alteraciones renales: se produce una retención acuosa que puede dar lugar a la aparición de edemas o hinchazón en las piernas.

Trastornos digestivos: consisten en anorexia, náuseas $\mathrm{y}$, ocasionalmente estreñimiento (Martín, 2017,p. 1).

Tabla 9. Sabe cómo alimentar a los niños para prevenir la anemia

\begin{tabular}{lcc}
\hline Descripción & Cantidad & $\%$ \\
\hline Sí & 24 & 34,29 \\
No & 46 & 65,71 \\
\hline Total & 70 & 100 \\
\hline
\end{tabular}

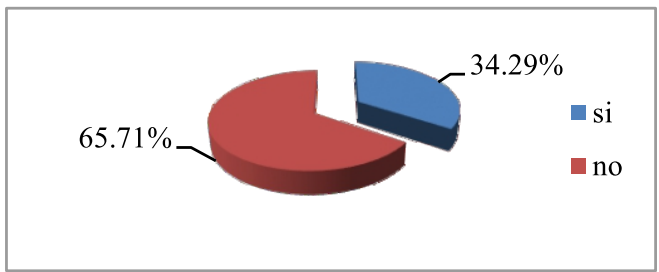

Figura 9. Sabe cómo alimentar a los niños para prevenir la anemia

El $65,71 \%$ dice no saber cómo alimentar a sus hijos para prevenir en ellos la anemia, esta situación resulta sumamente preocupante; sin embargo, es evidente que al conversar con las madres, estas no quieren mencionar que el problema se debe a la falta de recursos económicos, les da vergüenza manifestarlo, lo cual resulta bastante comprensible. En otros casos es porque lo que cultivan no es suficiente. Por otro lado, el $34,29 \%$ menciona que sí conoce la manera como alimentar a sus niños, y aunque esta respuesta reconforta, no representa a un grupo mayoritario; por esto se tiene que repensar en las políticas gubernamentales y de sector salud.

Tabla 10. Idioma de Personal de Salud

\begin{tabular}{|c|c|c|}
\hline Características & Cantidad & $\%$ \\
\hline Castellano & 60 & 85,71 \\
\hline Quechua & 10 & 14,29 \\
\hline Total & 70 & 100 \\
\hline \multirow[t]{2}{*}{$14.29 \%$} & \multirow{2}{*}{\multicolumn{2}{|c|}{$\begin{array}{l}\text { " castellano } \\
\text { quechua }\end{array}$}} \\
\hline & & \\
\hline & & \\
\hline
\end{tabular}

Figura 10. Idiomas que habla el personal de salud

Las madres encuestadas manifiestan que el personal del establecimiento de salud habla en castellano, lo cual se refleja en el $85,71 \%$ de casos. Como el idioma materno de las madres es el quechua, ellas manifiestan que aunque hablen también el castellano, este no es fluido; por tanto, el diálogo y entendimiento entre el personal de salud y los habitantes del distrito de Ccatcca son diferentes, si a ello se suma las tradiciones y costumbres distintas entonces es lógico que la comunicación presente dificultades mayores. De esto se deduce la necesidad de que el personal del centro de salud hable de manera fluida también el quechua; no obstante, solo el 14,29\% del personal lo hace.

La comunicación entre las personas es la forma que tenemos para ponernos en contacto y saber lo que el otro o los demás integrantes de la comunidad piensan, la falta de integración del personal con la comunidad no permite una comunicación adecuada. 
Además, aunque alguna parte del personal de los establecimientos de salud habla el quechua, no le da la entonación ni el significado contextual necesario para que la población pueda comprender los mensajes que el profesional de salud quiere transmitirles; asimismo, el personal contratado del lugar es para realizar actividades de menor nivel y no genera oportunidades para convertirse en transmisores de los conceptos médicos.

\section{DISCUSIÓN}

Contrastando la información, obtenida a través de las encuestas y la observación, con el análisis teórico de la investigación se puede colegir que posterior a las campañas de salud del año 2015, los resultados han sido adversos. No es difícil advertir que si en el centro de salud solo el $15 \%$ del personal habla el quechua, en tanto el $85 \%$ de las madres que son atendidas se expresa en quechua, los resultados no podrían ser distintos. Una información publicada en el boletín 34 del gobierno regional de Cusco, señala que el $65 \%$ de niños de la provincia de Quispicanchis tiene anemia (Gobierno regional de Cusco, 2017). Porcentaje suficiente para señalar que los programas de Salud Pública han fracasado. En alusión estricta a la teoría de la comunicación intercultural, a criterio de Rizo García, existen divergencias entre multiculturalidad e interculturalidad, teniendo estos últimos aspectos significativos que pueden trascender en otras actividades más allá de su propia actividad lingüística (Rizo García, 2013). Espinoza, citado por Rodríguez Ledesma (2008), señala que dichos conceptos deben partir desde el respeto y el reconocimiento de la existencia de diversidad hasta la necesidad de pugnar por crear las condiciones de igualdad bajo esa diversidad. Sin embargo, puede notarse claramente la falta de respeto por parte de una institución hacia una población quechua hablante, y esto se pone de manifiesto en el hecho de no considerar otro idioma distinto al castellano como elemento trascendente en la comunicación; por lo que sus efectos negativos trascienden en la salud de los niños de Ccatcca, y probablemente también en la educación, la economía y la propia calidad de vida de su población.
Nuestro país posee oficialmente varios idiomas, entre ellos, el español hablado por la mayoría $(83,92 \%)$, el quechua, incluidas sus variantes $(13,21 \%)$ y el aymara $(1,76 \%)$ utilizado por una minoría del total de la población peruana. Sumado a ello existen más de 40 lenguas aborígenes habladas por habitantes de la sierra y la selva. Si nos centramos solo en las personas quechua hablantes estaríamos haciendo referencia a un total de 3262137 personas en todo el territorio peruano, cifra que representa también la mitad de estas en toda Latinoamérica (UNICEF, 2009).

La región Cusco cuenta con el mayor número de quechua hablantes del país, siendo su incidencia mayor en distritos que a su vez son catalogados a nivel nacional como zonas de pobreza y de pobreza extrema, donde los servicios básicos como salud, educación, agua potable, desagüe, energía, transporte, entre otros, son extremadamente precarios en algunos casos y en otros no existen.

Acorde con los análisis anteriores, el distrito de Ccatcca, con un total de 19798 habitantes, solo tiene una población alfabeta del $12 \%$ mayor de 15 años. Por ende, se requiere de un tratamiento especial en términos de difusión de la nutrición infantil. Tomando en cuenta que la producción agraria del distrito es en su mayoría para autoconsumo y peor aún si el $85 \%$ de su población es quechua hablante, podríamos atrevernos a afirmar que todo programa social que no se estructure en base a dichas características está destinado a no tener resultados óptimos, es el caso de la actual política de salud pública en el Perú.

Las experiencias en torno a la inclusión de sectores rurales en la problemática de la salud deben ser mayores que los mismos programas de salud pública, donde se introduce un conjunto de requerimientos a la problemática; por lo que sería necesario mejores y mayores talleres de incorporación social, donde se identifiquen los derechos y deberes de los habitantes, considerando a la comunicación como elemento concomitante que coadyuva a esa inclusión, formando parte de un programa integral (Martínez Flores \& Granda, 2007). En 
programas de salud pública, tal como hemos apreciado en la investigación, se hacen despliegues infructuosos en papelería, sin tomar en cuenta que la comunicación intercultural requiere de más interacción personal (Pech, C., Rizo, M. \& Romeu, V., 2008). En tal sentido las propuestas de salud pública, que no asumen estas consideraciones, se permiten lapidar fondos estatales en programas que terminan con resultados ineficaces.

\section{CONCLUSIONES}

Ccatcca se ubica en una zona rural eminentemente agropecuaria y entre sus actividades económicas tiene predominio la agricultura, cuyos productos en su mayoría son de autoconsumo. Cuenta con una población quechua hablante del $84 \% \mathrm{y}$, en proyección al 2017 , con una población infantil del $65 \%$ con desnutrición infantil; por tanto, se hace sumamente necesario replantear los programas de Salud Pública. A lo largo de esta investigación se ha podido demostrar que dichos programas no tomaron en cuenta las barreras de comunicación intercultural, principalmente el idioma y todas sus connotaciones; el balance teórico nos demuestra que la comunicación intercultural puede trascender más allá de su propia actividad lingüística, influyendo en la calidad de vida de los niños, así también es importante recordar que las condiciones de respeto por la diversidad cultural generan condiciones de equidad.

Otros dos aspectos relevantes de la investigación son: Primero, el embarazo temprano de las mujeres en Ccatcca repercute en una inmadurez de las madres y su deficiente información en la alimentación de los niños; segundo, urge el desarrollo de las campañas de Salud Pública cuyas formas de información requieren un tratamiento intercultural en sus mensajes, por lo que el personal de salud, debe fortalecer sus conocimientos en la lengua quechua, de manera que la comunicación sea fluida y permita transmitir mejor las falencias en su alimentación, así también los cuidados y reconocimiento de enfermedades como la anemia.

\section{REFERENCIAS BIBLIOGRÁFICAS}

Cavero Carrasco, R. (2011). Interculturalidad y Globalización (Primera Edición). Lima, Perú: DSG Vargas S:R:L.

Clifor, G. (2003). La Interpretación de las Culturas. Barcelona, España: Gedisa Editorial.

Cusco, G. R. (22-27 de agosto de 2017). Boletín epidemiológico. XVII. (T. E. Salcedo, Ed.) Cusco, Perú. $\mathrm{O} b \mathrm{t}$ e $\mathrm{n}$ i d o d e www.diresacusco.gob.pe/inteligenc ia/epidemiologia/boletines/2017/34 . pdf

Degregori Caso, C. I. (2013). Del Mito del Inkarri al mito del progreso: Migración y cambios culturales (Primera Edición, Vol. III). (P. S. Agüero, Ed.) Lima, Perú: Instituto de Estudios Peruanos.Fierro, V. M. (agosto - octubre de 2000). Razón y Palabra. (O. Islas, Editor) Recuperado el 09 de 05 de 2017, de www.razonypalabra.org.mx : http://www.razonypalabra.org.mx/a nteriores/n19/19 vhernandez.html

Hall, S. (2010). Sin Garantías:Trayectorias Problemáticas en Estudios Culturales (Primera Edición). Lima, Perú: Instituto de Estudios Peruanos.Martín, E. (24 de octubre de 2017). Revista de Salud y B i enestar. Obtenido de https://www.webconsultas.com/ane mia/anemia-265

Martínez Flores , A. \& Granda, S. (2007). Curso de Formación Ciudadana Intercultural en el Programa de Educación Intercultural Bilingüe. Lima: Fondo Editorial de la PUCP.

Parra Crespo , M. F. (2010). Repositorio Digital de la Universidad de $\mathrm{Cu}$ e n c a. Obte n i d o d e http://dspace.ucuenca.edu.ec/handle /123456789/2983

Pech Salvador, Cynthia; Rizo García, Marta \& Romeu Aldaya Vivian. (2008). Manual de Comunicación Intercultural. México: Universidad Autónoma de la Ciudad de México.

RAE, R. A. (2014). Diccionario de la Real 
Académia Española. Madrid.

Rizo García, M. (15 de marzo de 2013). C O M U N I C A C I Ó N E INTERCULTURALIDAD. (G. M. México, Ed.) Recuperado el 23 de abril de 2017, de Global Media J o u r n a $1 \quad$ M é x i c o : http://www.redalyc.org/pdf/687/687 26424002.pdf

Rodríguez Ledesma, X. (2008). Una Historia desde y para la Interculturalidad (Ùnica ed.). México: Universidad Pedagógica Nacional.

Sánchez Arroba, M. (21 de diciembre de 2016). revistas.pucp.edu.pe/index.php/lexi s/article/download/5801/5793. $\mathrm{O} b \mathrm{t}$ e $\mathrm{n}$ i d o d e file://C:/Users/pc/Downloads/5801 -22361-1-PB.pdf

Tannen, D. (1999). La Cultura de la Polémica. Barcelona, España: PAIDOS.

UNICEF, F. (2009). Atlas Sociolinguístico de
Pueblos Indígenas en América Latina (Vol. 2). Cochabamba, Bolivia: UNICEF.

\section{ANEXO}

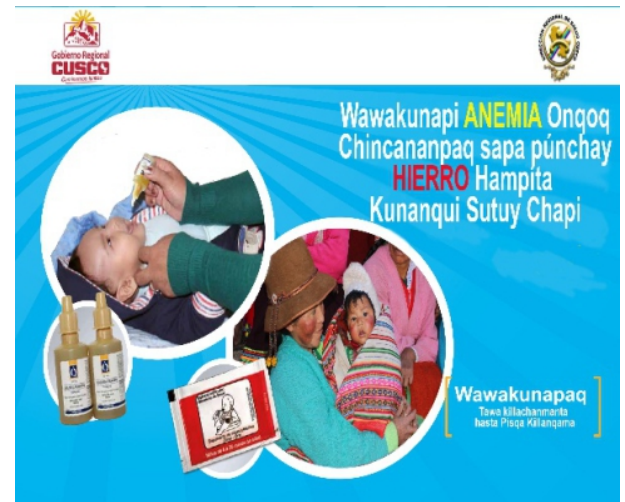

En la papelería de Salud Pública únicamente se utilizaron mensajes en quechua directos, no se da importancia a otros aspectos interculturales. 